\title{
Nonmetallic Inclusions in Steel - Origin, Estimation, Interpretation and Control
}

\author{
Alexander A. Kazakov ${ }^{1}$ \\ 1. Peter the Great Saint Petersburg Polytechnic University, St. Petersburg, Russia
}

An understanding of the origin of nonmetallic inclusions in steel, the estimation of their volume fraction, interpretation of their chemical composition and, finally, learning to control their formation processes at all stages of ladle treatment and casting up to final product requires an interdisciplinary knowledge of steel making theory and practice, quantitative metallography and microprobe analysis. Efforts to understand nonmetallic inclusions using all of these topics gives us the possibility to minimize the detrimental effects of deoxidation products, which are unavoidable in steel making processing. This knowledge may allow conversion of nonmetallic inclusions into a useful steel component; for example, to create a fine dendritic structure by inoculation of the melt or to control structure formation in the solid metal by promoting intergranular acicular ferrite precipitation on the nonmetallic inclusions as well as on the substrate.

Inclusions are classified as being either exogenous or indigenous according to their origin in the steel. The exogenous inclusions are entered into the melt from various external sources. The indigenous inclusions usually are naturally occurring steel deoxidation products formed during ladle treatment, casting and solidification.

According to their thermal nature, inclusions can be divided into the primary types formed under isothermal conditions of ladle treatment immediately after additions are introduced; or, secondary types formed during cooling of the liquid deoxidized steel to the liquidus temperature; or tertiary types formed in the solidifying steel. Quaternary type inclusions are formed in solid steel and they are usually carbonitrides of titanium, niobium and vanadium. The appearance of exogenous inclusions in steel is random, so their amount and location cannot be predicted or estimated. The volume fraction, composition, size and distribution pattern of indigenous inclusions can be predicted and accurately measured by quantitative metallography using light optical metallography or using the SEM-EDS microanalysis method, called automated feature analysis (AFA), to additionally evaluate the chemical composition.

Examples of the calculation of the solubility of isothermal surfaces of the components in the liquid steel, showing how the composition of nonmetallic inclusions change by varying the concentration of different deoxidizers in the steel, will be presented. It will be shown that for the simulation of the formation of nonmetallic inclusions in the liquid and solidified steel, the principle of local equilibrium thermodynamics can be used: the concentrations of elements around the growing inclusions are reaching their equilibrium concentrations immediately after the temperature of the molten steel begins decreasing. Simulation results for the formation of primary, secondary and tertiary nonmetallic inclusions in the liquid and solidifying steel for various deoxidation schemes with different amounts of deoxidizing elements will be demonstrated. Using the example of tertiary titanium oxycarbonitrides it will be shown that the chemical heterogeneity of inclusions across their section can be predicted. This information is useful to understand at what stage of solidification they were formed.

The equiaxed solidification of steel with nucleation upon titanium nitrides for steel inoculating is well known. The results of the simulation against the background of the real temperature curve, which allows estimation of the ratio of heterogeneous nucleation on substrates and of homogeneous nucleation centers during the formation of the dendritic structure; or, in other words, the simulation 
results that take into account the contribution of nitrides in dendrite formation will be presented [1]. The limitations to control nonmetallic inclusion size in the industrial molten steel will be shown. Thus, nanoscale inclusions are formed only by quenching the melt at a cooling rate unattainable in steel mass production. This conclusion is also proved by experimental studies with indigenous inclusions and with ultrafine exogenous particles injected into the melt.

A comparative analysis of the known methods of nonmetallic inclusions estimation in steel: ASTM E 1245, ASTM E 2283 , ASTM E 45 , DIN 50602 , EN 10247 , ISO 4967 , SEP 1572 , GOST R51685, GOST 801 , GOST 1778 will be reported. The advantages of using ASTM E 1245 for quantification of nonmetallic inclusions in steel based on the example of the implementation of this standard in the Thixomet Image Analyzer [2] will be demonstrated. Examples of the application of ASTM E 1245, not only to estimate the quality of the finished metal, but also to monitor the nonmetallic inclusions during secondary metallurgy, will be depicted. Unfortunately, none of the known standards contain nonmetallic inclusion classifications corresponding to modern methods of ladle treatment. Therefore, the use of SEM-EDS microanalysis method [3] could be a basis for the creation of such a classification.

The interpretation of the SEM-EDS microanalysis method results for nonmetallic inclusions is based upon: 1) determination of the composition of the oxide inclusions according to the elemental composition of the deoxidants and sulfur; 2) approving of the composition of the oxides based on the respective phase diagram; 3) evaluation of residual concentrations of elements in the steel, during which these oxides of approved composition are formed; and, 4) revealing the nature of the inclusions with approved composition using thermodynamic simulation.

A few examples of the practical use of thermodynamic simulation and interpretation of SEM-EDS microanalysis method results for solving specific problems of the steel industry will be described [4].

It will be shown that the formation of surface defects on cold-rolled sheets lies in violation of deoxidation technology, leading to the formation of hercynite $\left(\mathrm{FeO} \cdot \mathrm{Al}_{2} \mathrm{O}_{3}\right)$ inclusions which are not easily removed during ladle treatment because of the high adhesion to the molten steel. Practical recommendations for improving the deoxidization technology to eliminate such defects are approved in industry. The origin of nonmetallic inclusions in pipeline steels as calcium aluminates $\left(6 \mathrm{Al}_{2} \mathrm{O}_{3} \cdot \mathrm{CaO}, 2 \mathrm{Al}_{2} \mathrm{O}_{3} \cdot \mathrm{CaO}, \mathrm{CaO} \cdot \mathrm{Al}_{2} \mathrm{O}_{3}\right)$, spinel $\mathrm{MgO} \cdot \mathrm{Al}_{2} \mathrm{O}_{3}$, fayalite $\mathrm{FeO}_{\mathrm{x}} \mathrm{SiO}_{2}$ was discovered. It is shown how to make industrial production of clean steel, including control of calcium aluminate composition during deoxidation and modification of the molten steel.

The positive impact of nonmetallic inclusions on the mechanical properties of the finished metal, an example of the "oxide metallurgy," will be described. Instead of deoxidizing the steel with aluminum, if the steel is deoxidized with titanium, deoxidation products are formed on the basis of titanium oxides. These inclusions are uniformly distributed throughout the volume of steel and serve as substrates for intergranular nucleation of acicular ferrite and provide perfect mechanical properties of the steel. Similar processes, which occur in welding, are also described [5].

References:

[1] A.A. Kazakov et al, Bulletin of the USSR AS. Metals, 3, (1989) p.60.

[2] A.A. Kazakov, D. Kiselev, Microscopy \& Microanalysis, 21 (2015), p. 457.

[3] A.A. Kazakov et al, Microscopy \& Microanalysis, 21 (2015), p.1755.

[4] A.A. Kazakov et al, CIS Iron and Steel Review, 1-2 (2007), p.7.

[5] A.A. Kazakov et al, Microscopy \& Microanalysis, 21 (2015) p. 1749. 